\title{
Four Years of Altering Neurological Symptoms: A Rare Case of a Massive Left Ventricular Thrombus in the Absence of Any Symptomatic Cardiac Disease
}

\author{
Payam Akhyari $^{1}$, Hiroyuki Kamiya ${ }^{1}$, Artur Lichtenberg ${ }^{1}$, Axel Haverich ${ }^{2}$, Malakh L. Shrestha ${ }^{2}$ \\ ${ }^{1}$ Department of Cardiothoracic, Transplantation and Vascular Surgery, Hannover Medical School, Hannover, Germany; ${ }^{2}$ Department \\ of Cardiovascular Surgery, Duesseldorf University Hospital, Duesseldorf, Germany. \\ Email: Payam.akhyari@med.uni-duesseldorf.de
}

Received December $14^{\text {th }}$, 2011; revised January $15^{\text {th }}$, 2012; accepted February $4^{\text {th }}$, 2012

\begin{abstract}
Cerebral Thrombemboli with left ventricular origin are occasionally seen in patients with post-infarction left ventricular aneurysm or dilatative cardiomyopathy of non-ischemic cause. Freedom of medical history of cardiac disease and corresponding symptoms may delay the proper diagnosis, particularly in patients with distinct neurological symptoms and normal findings in electrocardiogram or cerebral MRI. We report on a rare case of long standing neurological symptoms and late diagnosis of a left ventricular thrombus without clinical symptoms or medical history of cardiac disease. The patient underwent a thrombus extraction via left ventricular apical approach. He was discharged from hospital after an uneventful course in our clinic with remaining mild neurological symptoms that were partially recurrent under physiotherapy and logopedic therapy in the course of the following two years. An echocardiographical evaluation of cardiac function and exclusion of a cardiac source of emboli as a first line diagnostic tool may have led to an early diagnosis. Therefore, it should be implemented in the routine examination, independent of cardiac history or present cardiac symptoms.
\end{abstract}

Keywords: Cardiac Surgery; Left Ventricular Thrombus; Cerebral Thrombembolism; Echocardiography

\section{Introduction}

Cerebral Thrombemboli with left ventricular origin are occasionally seen in patients with post-infarction left ventricular aneurysm or dilatative cardiomyopathy of nonischemic cause [1,2]. Most of these patients have a history of angina or heart failure symptoms that is under medical or surgical treatment including pharmacological regimen and interventional or surgical therapy. Complications resulting from cerebral thrombemboli include transient ischemic attacks (TIA), prolonged ischemic neurological deficits or a manifest apoplexy [3]. The associated symptoms vary from regional sensory impairment to complete loss of motor function, requiring immediate initiation of further diagnostic steps and therapy $[4,5]$.

\section{Case Report}

A 56 year old male patient was presented to the emergency unit of a community hospital for partial aphasia and impairment of fine motor movement. He reported suffering from nonspecific intermittent paraesthesia and dysesthesia in both forearms and hands, as well as both lower extremities for about four years. After a neuronlogical consultation, oral vitamins were prescribed.

However, symptoms remained with altering intensity for another two years and the patient occasionally visited his general practitioner for his complaints.

Subsequent hospitalization for neurological workup in a community clinic brought no significant finding. A cranial and cervical MRI showed no pathological finding at that time. Laboratory blood and liquor tests brought no sign of a chronic inflammatory process or infection. After discharge under oral Vitamin B complex in the following 18 months the patient was sporadically free of symptoms, but most of the time numbness and paraesthesia of right upper extremity were present. At this time, a thrombembolic pathology as the main underlying cause of the observed symptoms was not anticipated, likewise not anticoagulation or platelet inhibition medication was initiated. About six months prior to admission to our clinic he experienced right facial paraesthesia and visual impairment of the right eye, including partial loss of vision and occurrence of yellow spots. These symptoms were neglected by the patient, who meanwhile suggested a psychological cause underlying his complaints. Adding 
to his symptoms, few weeks later he experienced a pronounced dysmetria and severe loss of spatial orientation. Finally, diminished cognitive power and word finding problems brought him to seek for another neurological consultation.

Multiple ischemic lesions typical of embolic character and of different age were detected on a subsequent cranial MRI that led to a cardiac work up. On a transthoracic echo a $60 \mathrm{~mm}$ long and $10-15 \mathrm{~mm}$ thick free floating mass was detected within the left ventricular (LV) chamber with its base attached to the akinetic anteroseptal wall (Figure 1). After angiographic exclusion of coronary disease, the patient underwent an open heart surgical procedure where the LV thrombotic mass was recovered via a transmural approach from the heart apex under cardiopulmonary bypass (Figure 2). Histopathological evaluation revealed a thrombotic mass with varying stage of organization and no indication for malignancy.

After an uneventful postoperative course, the patient was discharged from the clinic on the $10^{\text {th }}$ postoperative day.

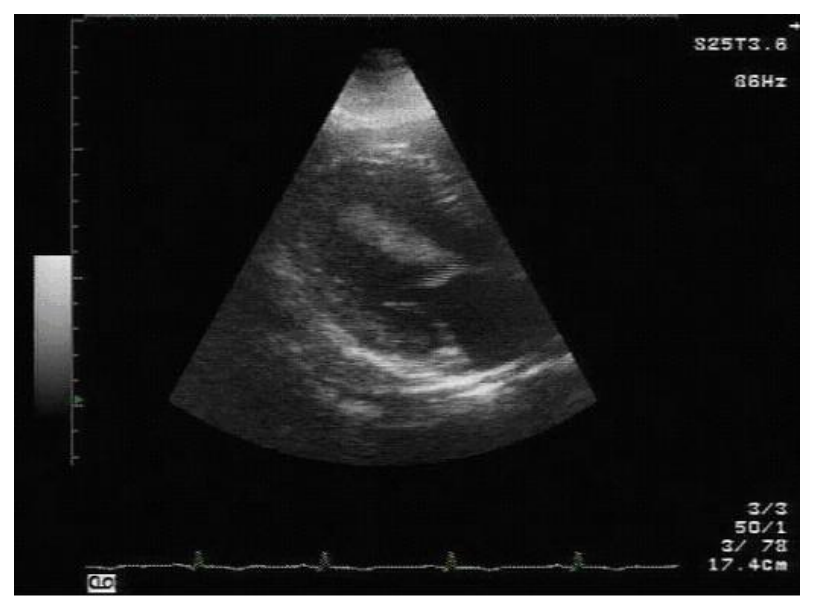

Figure 1. Massive left ventricular (LV) thrombus adherent to the anteriobasal wall. Echocardiography. LV, left ventricular cavity; *, thrombus.

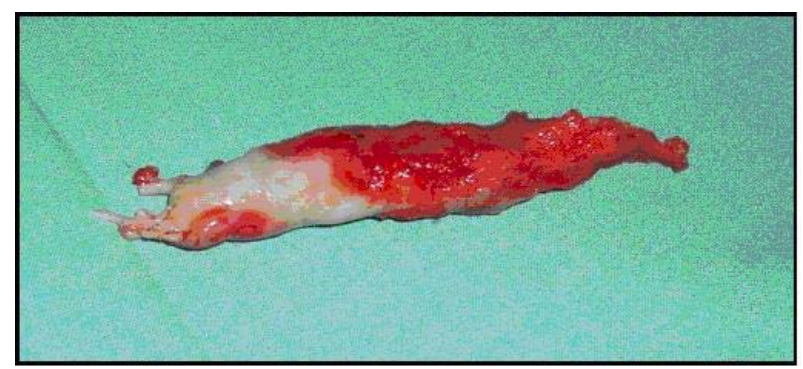

Figure 2. Macroscopical aspect of the thrombus after surgical removal. Organized and more recent thrombotic aspects can be detected.

\section{Summary and Conclusions}

An intracardiac source of emboli may be in first line suggested in patients with prediagnosed cardiac disease. Also patients presenting with severe neurological symptoms are prone to a broad diagnostic work up including cardiac evaluation. However, in patients with minor sporadic neurological complaints and no cardiac symptoms, arriving at the proper diagnosis may be more problematic.

Here, we report on a rare case of long term neurological symptoms of changing character and localization with late diagnosis of a free floating left ventricular thrombus. This case study shows that the absence of any symptoms typical of cardiac disease and a normal ECG finding might mislead the search for the underlying cause. The absence of ischemic lesions on the initial cMRI may be explained by a minimal size and extension of the cerebral injury at that early time. While earlier diagnostic workup has been profoundly mislead by this negative finding, the true cause remained undetected for more than four years. We conclude that echocardiographical evaluation of cardiac function and exclusion of a cardiac source of emboli is an effective and simple way to add important diagnostic data. Therefore, it should be implemented in the routine examination, independent of cardiac history or present cardiac symptoms.

\section{REFERENCES}

[1] E. Loh, M. S. Sutton, C. C. Wun, J. L. Rouleau, G. C. Flaker, S. S. Gottlieb, et al., "Ventricular Dysfunction and the Risk of Stroke after Myocardial Infarction," New England Journal of Medicine, Vol. 336, No. 4, 1997, pp. 251-257. doi:10.1056/NEJM199701233360403

[2] J. R. Stratton, J. W. Nemanic, K. A. Johannessen and J. D. Resnick, "Fate of Left Ventricular Thrombi in Patients with Remote Myocardial Infarction or Idiopathic Cardiomyopathy,” Circulation, Vol. 78, No. 6, 1988, pp. 13881393. doi:10.1161/01.CIR.78.6.1388

[3] G. W. Albers, L. R. Caplan, J. D. Easton, P. B. Fayad, J. P. Mohr, J. L. Saver and D. G. Sherman, "Transient Ischemic Attack-Proposal for a New Definition,” New England Journal of Medicine, Vol. 347, No. 21, 2002, pp. 1713-1716. doi:10.1056/NEJMsb020987

[4] S. C. Johnston, "Clinical Practice. Transient Ischemic Attack,” New England Journal of Medicine, Vol. 347, No. 21, 2002, pp. 1687-1692. doi:10.1056/NEJMcp020891

[5] H. P. Adams Jr., B. H. Bendixen, L. J. Kappelle, J. Biller, B. B. Love, D. L. Gordon and E. E. Marsh III, "Classification of Subtype of Acute Ischemic Stroke. Definitions for Use in a Multicenter Clinical Trial. TOAST. Trial of Org 10172 in Acute Stroke Treatment,” Stroke, Vol. 24, No. 1, 1993, pp. 35-41. doi.10.1161/01.STR.24.1.35 